\title{
DESEMPENHO DE SEMENTES DE ALGODOEIRO SUBMETIDAS A DIFERENTES TIPOS DE ESTRESSES ${ }^{1}$
}

\author{
FABIO MATTIONI², MARIA CRISTINA DE FIGUEIREDO E ALBUQUERQUE 3 , \\ ELISABETH APARECIDA FURTADO DE MENDONÇA ${ }^{4}$
}

\begin{abstract}
RESUMO - As variações de temperatura e umidade durante o período de emergência das plântulas são as condições de estresses mais importantes e que apresentam maiores riscos para a emergência do algodoeiro. Os objetivos deste trabalho foram verificar a influência do tipo de estresse no desempenho germinativo de sementes de algodoeiro e determinar a eficiência do teste de germinação, a baixa temperatura. Foram utilizados cinco lotes de sementes, submetidos previamente a determinação do grau de umidade, germinação e vigor (germinação a baixa temperatura). As sementes de cada lote foram submetidas a condições de estresses de temperatura de $18^{\circ} \mathrm{C}$ e $35^{\circ} \mathrm{C}$ e de disponibilidade hídrica de $40 \%$ e $80 \%$. Após a germinação em situação de estresse, foram avaliadas a porcentagem e velocidade de emergência de plântulas e o comprimento de raiz e de parte aérea. As avaliações dos comprimentos de raiz e de parte aérea a $18^{\circ} \mathrm{C}$ foram mais eficientes do que a porcentagem de emergência de plântulas para a diferenciação de lotes quanto ao potencial fisiológico. A exposição das sementes a estresses ocasionados pela alta disponibilidade hídrica e baixa temperatura proporciona menor desempenho germinativo dos lotes de sementes de algodoeiro e podem representar os maiores riscos durante a emergência das plântulas. $\mathrm{O}$ teste de germinação a baixa temperatura e eficiente para estimar o desempenho das plântulas em condições de baixa e alta temperatura e de baixa umidade.
\end{abstract}

Termos para indexação: Gossypium hirsutum, teste de vigor, temperatura, umidade.

\section{PERFORMANCE OF COTTON SEEDS SUBMITTED TO DIFFERENT TYPES OF STRESS}

\begin{abstract}
Temperature and moisture variations during the plantlet emergence period are the most important types of stress, representing the greatest hazards in cotton emergence. The objective of this study was to investigate the influence of type of stress on the germination performance of cotton seeds and determine germination test effectiveness at low temperature. Five seed lots were previously submitted to moisture content, germination, and vigor tests (germination at low temperature). The seeds from each lot were then submitted to temperature $\left(18^{\circ} \mathrm{C}\right.$ and $\left.35^{\circ} \mathrm{C}\right)$ and water availability $(40 \%$ and $80 \%$ ) stresses conditions. After germination was conducted under a stress situation, evaluations were made for plantlet emergence percentage and velocity, and root length and above-ground part length. Root length and above-ground part length at $18^{\circ} \mathrm{C}$ were more effective than plantlet emergence percentage in discriminating the seed lots with respect to their physiological potential. Seed exposure to stress produced by high water availability and low temperature cause reduced germination
\end{abstract}

${ }^{1}$ Submetido em 04/06/2008. Aceito para publicação em 09/01/2009.

${ }^{2}$ Eng. Agr., Doutorando do Programa de Pós Graduação em Agricultura Tropical, FAMEV/UFMT e bolsista FAPEMAT, fmattioni@yahoo.com.br

${ }^{3}$ Eng. Agr., Dr., Professora Associada, Programa de Pós Graduação em
Agricultura Tropical, FAMEV/UFMT, mcfa@ufmt.br

${ }^{4}$ Eng. Agr., Dr., Professora Adjunto, Programa de Programa de Pós Graduação em Agricultura Tropical, FAMEV/UFMT, beth@ufmt.br 
performance of the cotton seed lots and may represent the greatest hazards during plantlet emergence. The germination test at low temperature is effective to estimate plantlet performance under low and high temperature and low moisture conditions.

Index terms: Gossypium hirsutum, vigor test, temperature, moisture.

\section{INTRODUÇÃO}

As técnicas de produção, armazenamento, deslintamento e tratamento de sementes de algodoeiro melhoraram significativamente na última década, porém o mesmo avanço não ocorreu com os testes para determinação do potencial fisiológico das sementes para estimar o desenvolvimento de lotes comerciais no campo, principalmente quando as condições ambientais no momento da semeadura são menos favoráveis.

Entre os principais estresses que ocorrem durante o período de semeadura do algodoeiro, nas condições em que ele é cultivado no Brasil, as variações de temperatura e de umidade durante o período de emergência das plântulas são os mais relevantes e, comumente, os que apresentam os maiores riscos para o estabelecimento normal da cultura.

As pesquisas em tecnologia de sementes vêm concentrando esforços para padronizar testes de vigor que reúnam informações mais seguras para diferenciar o potencial fisiológico de lotes de sementes (Marcos Filho, 2005). Sobre esse aspecto, Albuquerque (2000) destacou que a semeadura de lotes com menor potencial fisiológico torna-se pouco interessante, pois esses apresentam menor resistência às condições de estresses ambientais.

Para a cultura do algodoeiro, o teste de germinação a baixa temperatura tem apresentado resultados mais expressivos para diferenciar lotes de sementes mais vigorosos; esse teste tem sido bastante pesquisado nos Estados Unidos (AOSA, 1983) Em estudos realizados por Torres (1998) e Freitas et al. $(2000 ; 2002)$ também foram encontradas evidências significativas para sua utilização em cultivares de algodoeiro adaptadas as condições brasileiras.

Contudo, para que os testes de vigor realizados em laboratório apresentem resultados semelhantes aos obtidos após a semeadura em campo, é necessário que seja correlacionado o efeito de cada tipo de estresse com a emergência das plântulas (Albuquerque, 2000).

O objetivo deste trabalho foi o de verificar a influência do tipo de estresse no desempenho germinativo de lotes de sementes de algodoeiro, além de, determinar se o teste de germinação a baixa temperatura é eficiente para estimar o melhor desempenho germinativo das sementes, quando submetidas a um tipo específico de estresse, com possibilidade de ocorrência por ocasião da semeadura, germinação e emergência de plântulas.

\section{MATERIAL E MÉTODOS}

Os experimentos foram conduzidos no Laboratório de Sementes da Faculdade de Agronomia e Medicina Veterinária da Universidade Federal de Mato Grosso. Foram utilizados cinco lotes de sementes de algodoeiro, cultivar FMT 701, peneira $9 \mathrm{~mm}$, coletados em uma mesma unidade de beneficiamento, com padrões de germinação acima de $75 \%$, conforme estabelecido pela instrução normativa $n^{\circ} 25$, de 16 de dezembro de 2005 (Brasil, 2005).

Para avaliar o potencial fisiológico das sementes foram realizados os testes: germinação - as sementes foram colocadas para germinar em substrato de papel, $25^{\circ} \mathrm{C}$, sendo realizadas duas contagens das plântulas normais, aos quatro e dez dias após o início do teste (Brasil, 1992) e germinação a baixa temperatura - as sementes foram dispostas sobre papel toalha umedecidas com água em uma proporção equivalente a 2,5 vezes a massa do papel seco e, em seguida, as sementes foram levadas para câmara de germinação a $18^{\circ} \mathrm{C}$, em ausência de luz, durante sete dias, sendo que na avaliação foram consideradas vigorosas as plântulas que atingiram $4 \mathrm{~cm}$ de comprimento (Dias e Alvarenga, 1999). Para que não houvesse interferência da quantidade de água presente nas sementes foi verificado o grau de umidade com duas subamostras de $5 \mathrm{~g}$ em estufa, tipo convecção gravitacional, a $105^{\circ} \mathrm{C} \pm 3^{\circ} \mathrm{C}$ por 24 horas (Brasil, 1992).

Os tipos de estresse, hídrico e de temperatura, foram determinados levando-se em consideração a possibilidade de ocorrência desses no momento da semeadura, germinação de sementes e emergência de plântulas em situações normais para as regiões de cultivo do algodoeiro no Brasil. Como condições ótimas para germinação foram adotadas a 
temperatura de $25^{\circ} \mathrm{C}$ e a capacidade de retenção de água no substrato de $60 \%$ (Brasil, 1992). Os tratamentos foram divididos em alta temperatura - as sementes foram colocadas para germinar em capacidade de retenção de $60 \%$ de umidade e levadas para câmara de germinação, regulada a $35^{\circ} \mathrm{C}$; baixa temperatura - capacidade de retenção de $60 \%$ e $18^{\circ} \mathrm{C}$; alta disponibilidade hídrica - capacidade de retenção de $80 \%$ e $25^{\circ} \mathrm{C}$ e baixa disponibilidade hídrica capacidade de retenção de $40 \%$ e $25^{\circ} \mathrm{C}$.

A capacidade de retenção de água foi calculada conforme a metodologia proposta por Brasil (1992). A semeadura foi realizada em areia disposta em caixas plásticas à profundidade de $2 \mathrm{~cm}$. As caixas foram colocadas em câmara de germinação regulada com fotoperíodo de 8 horas de luz e 16 horas de escuro. Para cada tratamento foram utilizadas quatro subamostras de 50 sementes. Para que não ocorresse a interferência de fungos patogênicos, as sementes foram tratadas, em todos os testes, com fungicida Carboxin $200 \mathrm{~g} / \mathrm{L}$ + Thiran $200 \mathrm{~g} / \mathrm{L}$ na dose equivalente a $400 \mathrm{~mL}$ para $100 \mathrm{~kg}$ de sementes.

Após 10 dias da semeadura foi avaliada a emergência de plântulas - foram computadas todas as plântulas emergidas, adotando-se como critério a completa expansão das folhas cotiledonares e o resultado foi transformado em porcentagem de emergência; índice de velocidade de emergência - foi calculado com base em dados provenientes de contagens diárias, de acordo com a fórmula de Maguire (1962); comprimento de raiz e de parte aérea - foi determinado utilizando-se um paquímetro, medindo-se a raiz, parte aérea e plântula, o resultado foi expresso em milímetros.

Os dados obtidos nos testes de caracterização inicial e de estresses foram submetidos à análise de variância e as médias foram comparadas pelo teste de Scott-Knott, a 5\% de probabilidade. Os valores obtidos em porcentagem foram transformados em arco seno $\sqrt{x / 100}$. As correlações entre os testes preliminares e os testes de estresses foram estimadas pelo coeficiente de correlação de Pearson. Foi utilizado para análise estatística, o programa estatístico SAEG da Universidade Federal de Viçosa-Viçosa/MG.

\section{RESULTADOS E DISCUSSÃO}

Na Tabela 1 encontram-se os resultados médios da qualidade inicial dos lotes de sementes de algodoeiro. Pelo teste de germinação foi verificado que o lote 4 apresentou o menor potencial fisiológico, enquanto que os demais lotes foram agrupados em um mesmo nível de qualidade fisiológica. Os lotes 3 e 4, pelo teste de germinação a baixa temperatura, apresentaram potencial fisiológico inferior, diferenciando-se significativamente dos demais.

TABELA 1. Qualidade inicial de cinco lotes de sementes de algodoeiro FMT 701, Cuiabá, MT, 2006.

\begin{tabular}{cccc}
\hline Lotes & TA & TG & TGBT \\
\cline { 2 - 4 } & \multicolumn{3}{c}{$(\%)$} \\
\hline 1 & 8,6 & $95 \mathrm{a}$ & $75 \mathrm{a}$ \\
2 & 8,5 & $97 \mathrm{a}$ & $77 \mathrm{a}$ \\
3 & 7,9 & $96 \mathrm{a}$ & $65 \mathrm{~b}$ \\
4 & 8,4 & $84 \mathrm{~b}$ & $51 \mathrm{~b}$ \\
5 & 8,3 & $97 \mathrm{a}$ & $86 \mathrm{a}$ \\
\hline
\end{tabular}

Médias dentro de cada coluna seguidas da mesma letra não diferem entre si pelo teste de Scott-Knott a 5\% de probabilidade. Teor de água (TA), teste de germinação (TG), teste de germinação a baixa temperatura (TGBT).

Comparando os resultados dos testes, verificou-se que a germinação foi inferior após as sementes serem submetidas ao teste de germinação a baixa temperatura. Esse teste, classificado como de estresse (AOSA, 1983; Dias e Alvarenga, 1999), avalia a resposta das sementes expostas a baixas temperaturas (Marcos Filho, 2005). Baseado nos resultados desse teste pode-se tomar decisões quanto ao potencial de armazenamento ou de semeadura de lotes de sementes, como sugerido por Freitas et al. (2002) e Baskin (2001).

Quando os lotes foram submetidos a diferentes condições de estresse hídrico (Tabela 2), foi verificado efeito significativo desse fator na porcentagem e índice de velocidade de emergência de plântulas a $80 \%$ de retenção de água. Os lotes 3 e 5 apresentaram menor porcentagem de emergência de plântulas nesse potencial hídrico. Por outro lado, o índice de velocidade de emergência foi menor em todos os lotes, exceto para o lote 4. Para Bewley e Black (1994), a água é o fator que exerce maior influência sobre o processo de germinação de sementes; essas apresentam modificações fisiológicas quando cultivadas em deficiência de oxigênio, ou ainda, em condições de alta umidade (Souza et al., 2001).

Quando foram analisados os comprimentos de raiz e de parte aérea, foi observada maior diferenciação entre os lotes, sendo que o lote 4 apresentou o pior desempenho quando em situação de deficiência hídrica (40\%), com menor desenvolvimento de raiz. Também, quando as sementes 
foram semeadas em condições favoráveis $(60 \%)$, às do lote 4 formaram plântulas com menor comprimento de parte aérea. Quando o estresse foi devido à alta umidade $(80 \%)$, não foram verificadas variações no comprimento de raiz e de parte aérea. Piana (1994) também observou que a deficiência hídrica retardou o desenvolvimento das plântulas durante a germinação de sementes de milho, e que tal fato estaria associado ao atraso da divisão e elongação celular.

TABELA 2. Características de cinco lotes de sementes de algodoeiro FMT 701 submetidas a diferentes condições de umidade. Cuiabá, MT, 2006.

\begin{tabular}{ccccccccccccc}
\hline \multirow{2}{*}{ Lotes } & \multicolumn{3}{c}{ PE (\%) } & \multicolumn{3}{c}{ IVE } & \multicolumn{3}{c}{ CR (cm) } & \multicolumn{3}{c}{ CPA (cm) } \\
\cline { 2 - 13 } & $40 \%$ & $60 \%$ & $80 \%$ & $40 \%$ & $60 \%$ & $80 \%$ & $40 \%$ & $60 \%$ & $80 \%$ & $40 \%$ & $60 \%$ & $80 \%$ \\
\hline 1 & $98 \mathrm{aA}$ & $99 \mathrm{aA}$ & $95 \mathrm{aA}$ & $10,2 \mathrm{aA}$ & $10,1 \mathrm{aA}$ & $8,8 \mathrm{bB}$ & $113,4 \mathrm{aA}$ & $69,2 \mathrm{bB}$ & $45,6 \mathrm{aC}$ & $70,1 \mathrm{bA}$ & $67,2 \mathrm{aA}$ & $57,7 \mathrm{aB}$ \\
2 & $98 \mathrm{aA}$ & $96 \mathrm{aA}$ & $96 \mathrm{aA}$ & $9,9 \mathrm{aA}$ & $10,4 \mathrm{aA}$ & $9,1 \mathrm{bB}$ & $100,5 \mathrm{bA}$ & $87,6 \mathrm{aB}$ & $41,7 \mathrm{aC}$ & $69,0 \mathrm{bA}$ & $64,8 \mathrm{aA}$ & $58,9 \mathrm{aB}$ \\
3 & $98 \mathrm{aA}$ & $99 \mathrm{aA}$ & $90 \mathrm{bB}$ & $10,5 \mathrm{aA}$ & $10,4 \mathrm{aA}$ & $8,2 \mathrm{bB}$ & $103,5 \mathrm{bA}$ & $87,0 \mathrm{aB}$ & $40,1 \mathrm{aC}$ & $69,5 \mathrm{bA}$ & $67,6 \mathrm{aA}$ & $58,5 \mathrm{aB}$ \\
4 & $99 \mathrm{aA}$ & $95 \mathrm{aA}$ & $96 \mathrm{aA}$ & $10,7 \mathrm{aA}$ & $9,9 \mathrm{aA}$ & $10,0 \mathrm{aA}$ & $89,1 \mathrm{cA}$ & $88,6 \mathrm{aA}$ & $41,1 \mathrm{aB}$ & $65,3 \mathrm{bA}$ & $59,0 \mathrm{bA}$ & $64,1 \mathrm{aA}$ \\
5 & $99 \mathrm{aA}$ & $98 \mathrm{aA}$ & $86 \mathrm{bB}$ & $10,4 \mathrm{aA}$ & $10,0 \mathrm{aA}$ & $8,6 \mathrm{bB}$ & $112,8 \mathrm{aA}$ & $97,0 \mathrm{aB}$ & $36,1 \mathrm{aC}$ & $79,9 \mathrm{aA}$ & $71,3 \mathrm{aB}$ & $58,9 \mathrm{aC}$ \\
\hline
\end{tabular}

Médias seguidas de mesmas letras, minúscula nas colunas e maiúscula nas linhas, na mesma variável, não diferem entre si pelo teste de Scott-Knott a 5\% de probabilidade. Porcentagem de emergência (PE), índice de velocidade de emergência (IVE), comprimento de raiz (CR) e comprimento de parte aérea (CPA)

Embora as sementes tenham apresentado uma faixa ampla de adaptabilidade à emergência em condições distintas de retenção hídrica, com variações pouco acentuadas em relação à testemunha (60\%), Tabela 2, o aumento da quantidade de água do substrato para $80 \%$ retardou a velocidade de emergência e proporcionou diminuição do crescimento radicular. Ainda que, para o crescimento de raiz, não tenham sido observadas diferenças significativas entre os lotes com o aumento da disponibilidade hídrica, foram percebidas diferenças acentuadas, em relação à testemunha (60\%), quando a quantidade de água do substrato aumentou para $80 \%$. O excesso de água provoca problemas devido a restrições à aeração e aos possíveis danos durante a embebição (Marcos Filho, 2005).

$\mathrm{O}$ algodoeiro demonstrou ser menos exigente em água, nos períodos iniciais de crescimento, visto que os resultados para o comprimento de raiz quando em baixa disponibilidade hídrica $(40 \%)$ foram significativamente superiores aos demais. Com o aumento da quantidade de água, para $60 \%$ e $80 \%$ da capacidade de retenção, houve decréscimo do comprimento de raiz; esse fato também foi observado para o comprimento de parte aérea, exceto para o lote 4. Os lotes 1 , 2 e 3 apresentaram resposta semelhante quando semeados a $40 \%$ e $60 \%$ da capacidade de retenção e maior comprimento de parte aérea nesses teores de água do que a $80 \%$. O lote 4 apresentou desempenho semelhante em todas as umidades e o lote 5 apresentou maiores comprimentos de parte aérea em todos os níveis de umidade.

Nos períodos iniciais de emergência, a demanda hídrica do algodoeiro é bem baixa, com taxa de consumo de água menor que uma polegada por dia (McWilliams, 2002) e apresenta modificações fisiológicas quando cultivadas em deficiência de oxigênio, ou seja, em condições de alta umidade (Souza et al., 2001).

Quando as sementes foram submetidas a diferentes condições de temperatura não foram observadas diferenças significativas entre os lotes na porcentagem e índice de velocidade de emergência de plântulas (Tabela 3), exceto para o índice de velocidade de emergência a $35^{\circ} \mathrm{C}$, para os lotes 4 e 5 , demonstrando que o algodoeiro possui adaptação à emergência sob uma faixa ampla de temperatura. Ocorreu, porém, maior velocidade inicial de emergência de plântulas nos períodos iniciais de embebição, quando as sementes foram expostas a $35^{\circ} \mathrm{C}$; o que não se observou a $18^{\circ} \mathrm{C}$. Essa maior velocidade de germinação em altas temperaturas também foi citada por Rosolem (1999), adaptando resultados obtidos por Wanjura e Buxton (1972), que observou que a elevação da temperatura provocou a redução do tempo para a emergência da raiz, em plântulas de algodoeiro.

Os comprimentos de raiz e parte aérea quando as sementes foram expostas a $18^{\circ} \mathrm{C}$ e $35^{\circ} \mathrm{C}$ variaram em função do lote. Os lotes 3 e 4 apresentaram menores comprimentos a $18^{\circ} \mathrm{C}$ e a $35^{\circ} \mathrm{C}$, quando comparados com os demais lotes. Porém, com a exposição das sementes a baixa temperatura 
$\left(18^{\circ} \mathrm{C}\right)$, o comprimento de parte aérea mostrou-se mais eficiente que o comprimento de raiz para diferenciar os lotes. O lote 5 apresentou resultado superior aos demais, os lotes 1 e 2 foram agrupados em nível intermediário e os lotes 3 e 4 apresentaram menor comprimento de parte aérea.

TABELA 3. Características de cinco lotes de sementes de algodoeiro FMT 701 submetidas a diferentes condições de temperatura. Cuiabá, MT, 2006.

\begin{tabular}{|c|c|c|c|c|c|c|c|c|c|c|c|c|}
\hline \multirow{2}{*}{ Lotes } & \multicolumn{3}{|c|}{ PE (\%) } & \multicolumn{3}{|c|}{ IVE } & \multicolumn{3}{|c|}{$\mathrm{CR}(\mathrm{cm})$} & \multicolumn{3}{|c|}{$\mathrm{CPA}(\mathrm{cm})$} \\
\hline & $18^{\circ} \mathrm{C}$ & $25^{\circ} \mathrm{C}$ & $35^{\circ} \mathrm{C}$ & $18^{\circ} \mathrm{C}$ & $25^{\circ} \mathrm{C}$ & $35^{\circ} \mathrm{C}$ & $18^{\circ} \mathrm{C}$ & $25^{\circ} \mathrm{C}$ & $35^{\circ} \mathrm{C}$ & $18^{\circ} \mathrm{C}$ & $25^{\circ} \mathrm{C}$ & $35^{\circ} \mathrm{C}$ \\
\hline 1 & $96 a A$ & $99 \mathrm{aA}$ & $98 \mathrm{aA}$ & $6,7 \mathrm{aC}$ & $10,1 \mathrm{aB}$ & $18,2 \mathrm{aA}$ & $44,8 \mathrm{aC}$ & $69,2 \mathrm{bB}$ & $80,6 \mathrm{aA}$ & $51,8 \mathrm{bC}$ & $67,2 \mathrm{aB}$ & $76,6 \mathrm{aA}$ \\
\hline 2 & $99 \mathrm{aA}$ & $96 \mathrm{aA}$ & $91 \mathrm{aA}$ & $7,5 \mathrm{aC}$ & $10,4 \mathrm{aB}$ & $17,1 \mathrm{aA}$ & 38,9aB & $87,5 \mathrm{aA}$ & $80,3 \mathrm{aA}$ & $49,6 \mathrm{bC}$ & $64,8 \mathrm{aB}$ & 73,7aA \\
\hline 3 & $93 \mathrm{aA}$ & $99 \mathrm{aA}$ & $100 \mathrm{aA}$ & $6,5 \mathrm{aC}$ & $10,4 \mathrm{aB}$ & $18,4 \mathrm{aA}$ & $36,0 \mathrm{bC}$ & $87,0 \mathrm{aB}$ & $75,8 \mathrm{bA}$ & $45,6 \mathrm{cB}$ & $67,5 \mathrm{aA}$ & 69,2 bA \\
\hline 4 & $93 \mathrm{aA}$ & $95 \mathrm{aA}$ & $97 \mathrm{aA}$ & $6,3 \mathrm{aC}$ & $9,9 \mathrm{aB}$ & $15,3 \mathrm{bA}$ & $28,3 \mathrm{bC}$ & $88,6 \mathrm{aA}$ & $70,6 \mathrm{bB}$ & $41,2 \mathrm{cC}$ & $59,7 \mathrm{bB}$ & $68,8 \mathrm{bA}$ \\
\hline 5 & $97 \mathrm{aA}$ & $98 \mathrm{aA}$ & $95 \mathrm{aA}$ & $6,9 \mathrm{aC}$ & $10,0 \mathrm{aB}$ & $15,8 \mathrm{bA}$ & $45,8 \mathrm{aB}$ & $97,0 \mathrm{aA}$ & $83,6 \mathrm{aA}$ & $61,4 \mathrm{aC}$ & $71,3 \mathrm{aB}$ & $80,6 \mathrm{aA}$ \\
\hline
\end{tabular}

Médias seguidas de mesmas letras, minúscula nas colunas e maiúscula nas linhas, na mesma variável, não diferem entre si pelo teste de Scott-Knott a 5\% de probabilidade. Porcentagem de emergência (PE), índice de velocidade de emergência (IVE), comprimento de raiz (CR) e comprimento de parte aérea (CPA)

De maneira geral, os efeitos de temperatura foram distintos (Tabela 3), enquanto que temperaturas maiores proporcionaram a aceleração da emergência de plântulas e maior desenvolvimento da parte aérea, temperaturas menores propiciaram retardamento da emergência e menores comprimentos de raiz e de parte aérea. Embora em baixas temperaturas não ocorra o comprometimento da germinação, foi verificado maior dificuldade para a emergência das plântulas. A temperatura exerce influência no processo germinativo, tanto por agir sobre a velocidade de absorção de água, como sobre as reações bioquímicas que determinam todo o processo; afetando, portanto, não só o total de germinação, como também a velocidade (Bewley e Black, 1994). A redução gradativa da temperatura, em função dos efeitos sobre a velocidade de embebição e de mobilização de reservas provoca decréscimo acentuado da velocidade de germinação (Marcos Filho, 2005). Nas temperaturas mais baixas, há redução dessa velocidade e alteração da uniformidade de emergência, expondo as sementes por um maior período a fatores adversos do ambiente. De forma contrária, nas temperaturas mais altas, ocorre maior velocidade de germinação (Carvalho e Nakagawa, 2000).

Não foram observadas correlações significativas entre os testes de germinação e germinação a baixa temperatura e os testes de porcentagem evelocidade de emergência deplântulas. Entretanto, quando se compararam os resultados dos testes de germinação e germinação a baixa temperatura (Tabela 1) com as características de crescimento (comprimento de raiz e de parte aérea) nos testes de estresse (Tabelas 2 e 3), foi possível observar valores significativos de $r$ (Tabela 4). As melhores estimativas para todas as características avaliadas para cada lote foram obtidas nos testes de temperatura a $18^{\circ} \mathrm{C}$, temperatura a $35^{\circ} \mathrm{C}$ e umidade de $40 \%$.

TABELA 4. Correlações lineares simples (r) entre resultados dos testes de vigor e de emergência deplântulas de algodão FMT 701, submetidas a diferentes tipos de estresses, Cuiabá, MT, 2006.

\begin{tabular}{cccc}
\hline \multirow{2}{*}{$\begin{array}{c}\text { Tipos de } \\
\text { estresse }\end{array}$} & \multicolumn{3}{c}{ Testes } \\
\cline { 2 - 4 } Vigor & \multicolumn{2}{c}{ Germinação } \\
\cline { 2 - 4 } Testemunha & CR & $0,0248^{\text {ns }}$ & $0,2260^{\text {ns }}$ \\
& CPA & $0,5723^{*}$ & $0,5713^{*}$ \\
\multirow{2}{*}{$18^{\circ} \mathrm{C}$} & $\mathrm{CR}$ & $0,6069^{*}$ & $0,7937^{*}$ \\
& $\mathrm{CPA}$ & $0,5882^{*}$ & $0,7881^{*}$ \\
$35^{\circ} \mathrm{C}$ & $\mathrm{CR}$ & $0,4534^{*}$ & $0,6290^{*}$ \\
& $\mathrm{CPA}$ & $0,3979^{*}$ & $0,6914^{*}$ \\
\multirow{2}{*}{$40 \%$} & $\mathrm{CR}$ & $0,7327^{*}$ & $0,7485^{*}$ \\
& $\mathrm{CPA}$ & $0,3817^{*}$ & $0,5288^{*}$ \\
$80 \%$ & $\mathrm{CR}$ & $-0,1331^{\text {ns }}$ & $-0,1483^{\text {ns }}$ \\
& $\mathrm{CPA}$ & $-0,5113^{*}$ & $-0,2550^{\text {ns }}$ \\
\hline
\end{tabular}

Teste de germinação (TG), teste de germinação a baixa temperatura (TGBT). Comprimento de raiz (CR), comprimento de parte aérea (CPA).

* significativo a $5 \%$ de probabilidade

ns - não significativo 
A tentativa de correlacionar testes de vigor com resultados promissores de emergência e desempenho de plantas é a busca de vários pesquisadores. Estudos neste sentido foram realizados mais recentemente por Torres (1998) e Freitas et al. (2000 e 2002) com a cultura do algodoeiro. Esses autores encontraram resultados promissores para os testes de envelhecimento acelerado e de frio sem terra e para o teste de germinação a baixa temperatura, respectivamente. Neste trabalho, o teste de germinação a baixa temperatura também foi constatado eficiente para avaliação de sementes de algodoeiro.

\section{CONCLUSÕES}

A alta disponibilidade hídrica e a baixa temperatura proporcionam menor desempenho germinativo em lotes de sementes de algodoeiro, além de afetar negativamente a emergência das plântulas.

O teste de germinação a baixa temperatura é eficiente para estimar o desempenho das plântulas em condições de baixa e alta temperatura e de baixa umidade.

Os comprimentos de raiz e de parte aérea a $18^{\circ} \mathrm{C}$ são avaliações eficientes na diferenciação de lotes quanto ao potencial fisiológico.

\section{REFERÊNCIAS}

ALBUQUERQUE, M.C.F. Desempenho germinativo de sementes de milho, soja, e girassol. I. Influência do tipo de estresse ambiental sobre a manifestação do nível de vigor das sementes. II. Testes de vigor mais eficientes para previsão de desempenho germinativo no campo sob diferentes tipos de estresse ambiental. 2000. 159f. Tese (Doutorado em Produção e Tecnologia de Sementes) Faculdade de Ciências Agrárias e Veterinárias, Universidade Estadual Paulista, Jaboticabal.

ASSOCIATION OF OFFICIAL SEED ANALISTS. Seed vigor testing handbook. [S.l.], 1983. 88p. Contribution $n^{\circ}$ 32 to the Handbook on Seed Testing

BASKIN, C. C. Three-day count as a vigor test for cottonseed. Seed Technology, v.23, n.1, p.81-84, 2001.

BEWLEY, J.D.; BLACK, M. Seeds: physiology of development and germination. New York: Plenum Press, 1994. 445p.

BRASIL. Ministério da Agricultura Pecuária e do Abastecimento. Instrução normativa $\mathbf{N}^{\circ}$ 25, de 16 de dezembro de 2005. Brasília, DF, 2005. Disponível em: $<$ http://extranet.agricultura.gov.br/sislegis-consulta/
consultarLegislacao.do>. Acesso em: 25 abr. 2007.

BRASIL. Ministério da Agricultura e Reforma Agrária. Regras para análises de sementes. Brasília, DF: SNAD/ CLAV, 1992. 365p.

CARVALHO, N. M.; NAKAGAWA, J. Sementes: ciência, tecnologia e produção. Jaboticabal: FUNEP, 2000. 588 p.

DIAS, D. C. F. S.; ALVARENGA, E. M. Teste de germinação a baixa temperatura. In: KRZYZANOWSKI, F. C.; VIEIRA, R. D.; FRANÇA NETO, J. B. (Ed.). Vigor de sementes: conceitos e testes. Londrina: ABRATES, 1999. p.7.1-7.4.

FREITAS, R. A.; DIAS, D. C. F. S.; REIS, M. S.; CECON, P.R. Correlação entre testes para avaliação da qualidade de sementes de algodão e a emergência das plântulas em campo. Revista Brasileira de Sementes, v.22, n.1, p.97-103, 2000.

FREITAS, R. A.; DIAS, D. C. F. S.; CECON, P.R.; REIS, M. S.; DIAS, L. A. S. Storability of cotton seeds predicted by vigour test. Seed Science and Technology, v.30, n.2, p.403-410, 2002.

McWILLIAMS, D.A. Producing quality cotton using irrigation management. In: IRRIGATION EFFICIENCY CONFERENCE, 7., New Mexico. Proceedings... New Mexico, 2002.

MAGUIRE, J.D. Speed of germination-aid in selection and evaluation for seedling emergence and vigor. Crop Science, v.2, n.1, p.176-177, 1962.

MARCOS FILHO. J. Fisiologia de sementes de plantas cultivadas. Piracicaba: FEALQ, 2005. 425p.

PIANA Z. Resposta de sementes de milho com diferentes níveis de vigor, à disponibilidade hídrica. 1994. 107f. Tese (Doutorado em Fitotecnia) - Escola Superior de Agricultura “Luiz de Queiroz”, Universidade de São Paulo, Piracicaba.

ROSOLEM. C. A. Ecofisiologia e manejo cultural do algodoeiro. In: MATO GROSSO - liderança e competitividade. Rondonópolis: Fundação MT, Campina Grande: EMBRAPA-CNPA, 1999.

SOUZA, J. G.; BELTRÃO, N. E. M.; SANTOS, J. W. Fisiologia e produtividade do algodoeiro em solo encharcado na fase de plântula. Pesquisa Agropecuária Brasileira, v.36, n.3, p.425-430, 2001.

TORRES, S. B. Comparação entre testes de vigor para avaliar a qualidade fisiológica de sementes de algodão. Revista Brasileira de Sementes, v.20, n.2, p.11-15, 1998.

WANJURA, D. F.; BUXTON, D. R. Water uptake and radicle emergence of cottonseed as affected by soil moisture and temperature. Agronomy Journal, v. 64, p. 427-431, 1972. 